\title{
Aneurysmal pinna - An unfamiliar entity
}

\author{
Deviprasad Dosemane', Meera N. Khadilkar², Hema Kini ${ }^{3}$, Fayis Anwar ${ }^{4}$ \\ ${ }^{1}$ Associate Professor, ${ }^{2}$ Assistant Professor, ${ }^{4}$ Senior Resident, Department of ENT \& HNS ${ }^{3}$ Professor, Department of Pathology, \\ Kasturba Medical College \\ Manipal Academy of Higher Education, Mangalore 575001, Karnataka, India
}

(Received: June $2020 \quad$ Revised: July $2020 \quad$ Accepted: July 2020)

Corresponding author: Meera N. Khadilkar. Email: meera.khadilkar@manipal.edu

\begin{abstract}
Lesions ranging from cysts to benign and malignant tumors can affect the pinna. The clinical appearance, duration, progression, and histopathology of the lesion are important in differentiating non-neoplastic conditions from neoplasms. We present a case report of an unusual nodular pinna lesion that was excised under local anaesthesia. Histopathology revealed cutaneous aneurysmal fibrous histiocytoma. Aneurysmal benign fibrous histiocytoma is an unusual variant of soft tissue tumor with low intermediate malignant potential, which has to be differentiated from other benign and malignant tumors.
\end{abstract}

Keywords: Benign fibrous histiocytoma; external ear; fibrous tissue neoplasms.

\section{INTRODUCTION}

$\mathrm{N}$ odules of the pinna range from epidermal cyst, chondrodermatitis nodularis chronica helicis, keloid, hemangioma, skin tag, seborrheic keratosis, pyogenic granuloma, tumors of the skin and adnexa like trichofolliculoma, trichoepithelioma and tumors of soft tissue like leiomyoma and dermatofibroma. The clinical appearance, duration and progression of the lesion are important in differentiating non-neoplastic conditions from neoplasms $(1,2)$. However, histomorphology is the corner stone of final diagnosis and especially so in evaluating borderline soft tissue neoplasms with potential for local recurrence and loco-regional spread (2-4).

\section{CASE REPORT}

A 64-year old woman presented to the outpatient department with a slow- growing swelling on the left pinna since one year. There was no associated pain or discharge. Examination showed a $1 \times 1 \mathrm{~cm}$. soft bluish swelling over the conchal aspect of left pinna, with a smooth vascular surface (Fig. 1). It was non-tender, without a local rise in temperature. There were no other abnormalities noted.

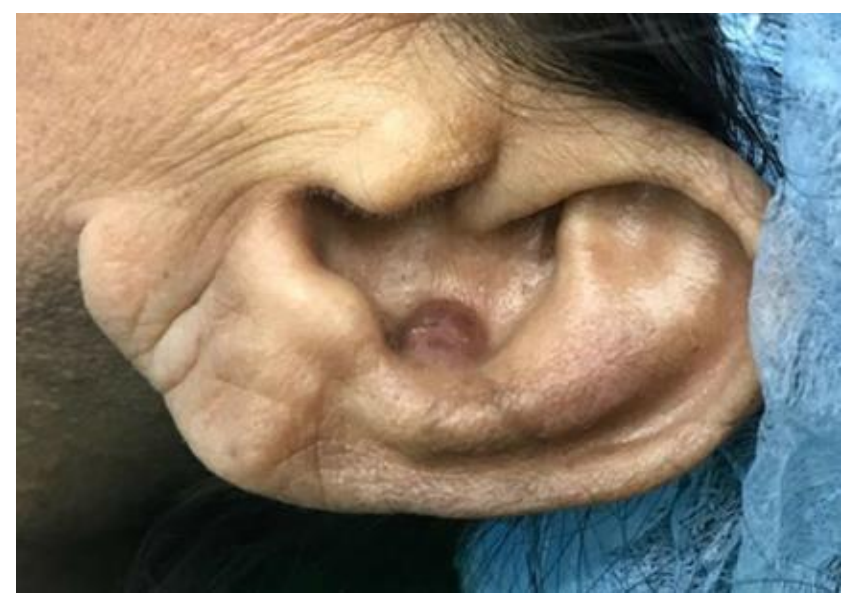

Fig. 1: Photograph showing lesion in left pinna
There were no palpable lymph nodes. The lesion was excised under local anaesthesia (Fig. 2) and the wound was sutured for healing by primary intention. Grossly, the excised margins appeared clear. Histopathology revealed skin with flattened dermis and a dermal based nodular tumor, composed of short fascicles, sheets and whorls of slender to plump spindle shaped cells, interdigitating with the dermal collagen at the periphery (Fig. 3) Haemorrhage with cystic change, histiocytes, multinucleated Touton giant cells and hyalinised vessels were noted. The lesion showed mitotic figures and intralesional, perilesional lymphocytic infiltrate. The deeper resected margin was involved by tumor. With the above features, a diagnosis of cutaneous aneurysmal fibrous histiocytoma was made. The patient came for follow-up for up to 6 months and was not found to have recurrence.

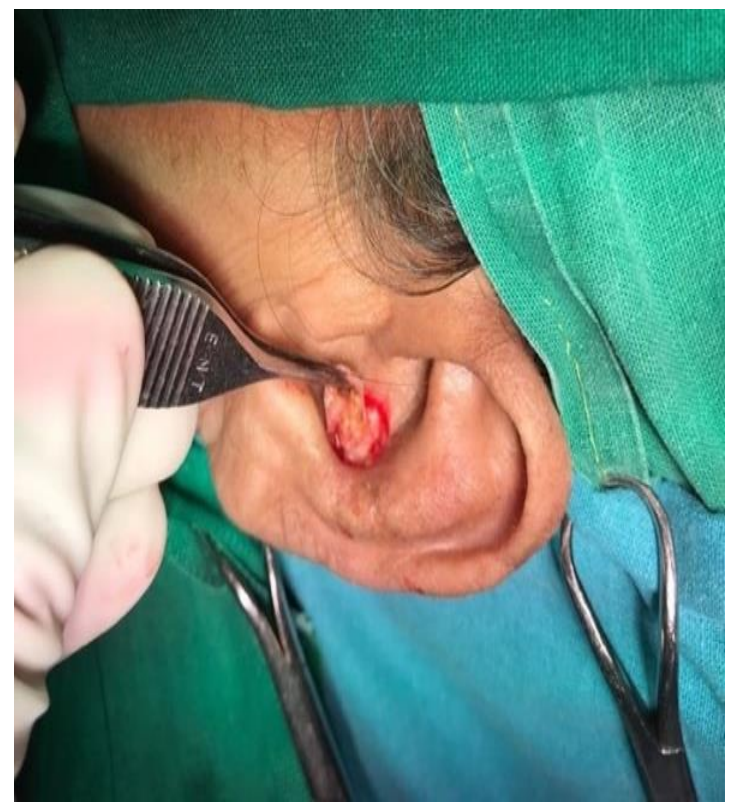

Fig. 2: Intraoperative photograph showing excision of the lesion 


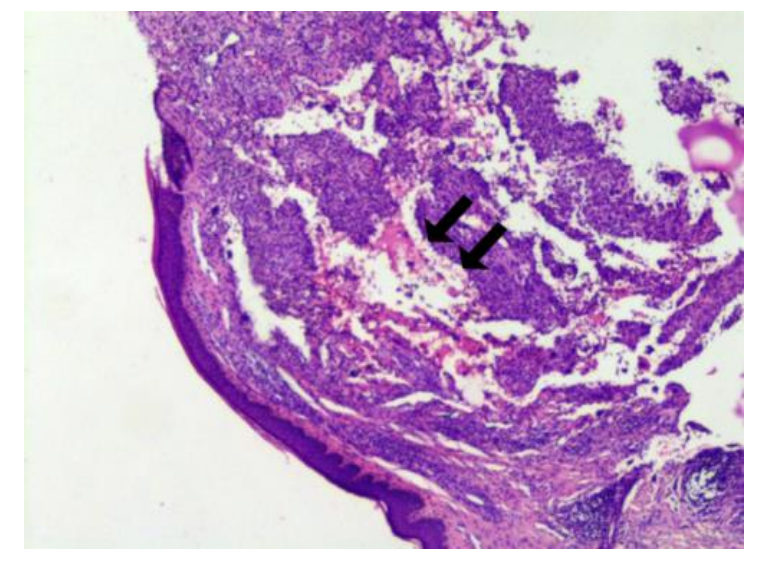

Fig. 3: Hematoxylin \& Eosin (H\&E) section [100x] Subepidermal lesion composed of irregular anastomosing bloodfilled spaces (double arrows) lined by septae.

\section{DISCUSSION}

The common differentials of skin and appendiceal lesions in the pinna include pilar cyst, trichoepithelioma and basal cell carcinoma. Trichoepithelioma is an unusual benign condition with single or multiple facial lesions after puberty, more often in females. The tumor cells form rudimentary hair follicles without leading to formation of actual hair shafts. Basal cell carcinoma is locally invasive, involving the basal layer of epidermis; common in elderly fair and dry skinned people, with constant exposure to sunlight. Pilar cysts are often familial and typically benign but with a locally aggressive, potential; seen in middle aged females (1).

Even though benign fibrous histiocytoma (BFH) is one of the most frequent soft tissue tumours of the skin, its occurrence in the pinna is rare. It is also known as dermatofibroma. Cutaneous FH arises in the reticular dermis but may quite often extend into the superficial subcutis (3). Aneurysmal Benign Fibrous Histiocytoma (ABFH) of the skin is a distinct variant with a reported incidence of $1.7 \%$ of all benign fibrous histiocytomas (4), first described by Santa Cruz and Kyriakos in 1981. The clinical and morphological features of this entity are not well recognized, resulting in diagnostic dilemma and confusion with other soft tissue tumor-like lesions. Clinically it has varied appearances and mimics benign and malignant vascular tumours or malignant melanoma resulting in diagnostic difficulties (2). It differs from ordinary benign fibrous histiocytoma by a greater than typical size, and rapid progression due to intra-lesional pigmentation and haemorrhage (4).

The incidence of ABFH is approximately $0.3 \%$ of all soft tissue neoplasms. It commonly presents as a solitary nodule on the limbs and extremities, also in sites of normal lymphoid tissue like the antecubital fossa, axilla, inguinal and supraclavicular regions. Few cases have been reported in the head and neck region (10\%) and trunk (4). It is commonly seen in young to middle aged adults with a slight female preponderance $(2,4)$. The chief histopathological criteria for diagnosis is the presence of blood-filled spaces mimicking artifactual clefts or cavernous vascular channels within a usual fibrous histiocytoma. Immunohistochemistry has not much of a noteworthy role in diagnosis. Tumour cells show diffuse positivity with vimentin only (5).

$\mathrm{ABFH}$ resembles fibrous tumors like fibroxanthoma in the cellular part of the tumor, and histopathological examination is obligatory for correct diagnosis (4). In the vascular part of the tumor, it has to be differentiated from angiomatoid malignant fibrous histiocytoma (MFH) and vascular tumors like nodular Kaposi's sarcoma and angiosarcoma. Angiomatoid MFH commonly affects younger age, arises in superficial subcutis, is well circumscribed and is composed of monomorphic round cells with prominent lymphohistiocytic infiltrate $(3,4)$. The dilated pseudovascular spaces resemble aneurysmal $\mathrm{FH}$; translocations $[\mathrm{t}(12 ; 16), \mathrm{t}(12 ; 22)$, or $\mathrm{t}(2 ; 22)]$ help in the diagnosis of AMFH (3).

Nodular Kaposi's sarcoma demonstrates CD34 positive cells lining slit-like spaces containing RBCs. Cutaneous angiosarcomas occur solely on face and scalp and have vascular spaces lined by atypical, mitotically active endothelial cells (4).

Focal necrosis in $\mathrm{ABFH}$ is not rare; this however does not determine clinical behaviour. No evidence of focal necrosis was noted in the present case. Mitotic activity may range from less than one to 13 per 10 high power field; it is comparable in primary, metastatic and recurrent ABFH. Obvious and/or atypical mitoses may be noted even in cases without recurrence or metastasis; hence, it is not a predictor of biologic potential of the tumour (3).

In a study by Doyle et al., 16 patients with morphologically benign cutaneous FH showed locoregional/ distant metastasis to internal organs. Surgical excision was performed for all the primary lesions, followed by adjuvant chemotherapy/ radiation/lymph node dissection for metastatic disease. Metastatic disease was identified between 0 and 180 months with a median of 17 months (3). In the present study, wide excision was not possible because of the location of the tumor in the pinna that would require reconstruction or result in a cosmetic deformity. The patient was regularly followed up for recurrence and loco-regional spread, since the tumor involved the deeper surgical margin.

Unclassified dermal sarcoma is another differential diagnosis to be considered in the evaluation of other variants of fibrous histiocytoma like cellular and metastasizing forms. Primary dermal sarcomas usually affect sun-damaged skin, are irregular, with diffuse atypia, fascicular growth pattern, frequent tumor necrosis, with vascular/perineural invasion (3). 
Metastasizing fibrous histiocytoma could be considered as sarcoma. Nevertheless, since they morphologically resemble conventional cutaneous histiocytomas without an aggressive course, any other diagnosis using the present diagnostic classifications is unlikely (3).

\section{CONCLUSION}

As aneurysmal benign fibrous histiocytoma is a clinical mimic of benign and malignant skin and soft tissue tumours, histopathological examination is imperative for diagnosis. It has a high local recurrence rate and is considered as a tumour of low malignant potential. Hence, complete excision with surgical clearance of margins is mandatory whenever possible.

\section{CONFLICT OF INTEREST}

Authors declare no conflict of interest.

\section{REFERENCES}

1. Karthikeyan, P., Paulraj, P. An unusual benign tumor of pinna - A case report. Indian J Otolaryngol Head Neck Surg. 2010; 62(2): 195-197.

2. López, V., Giner, F. Nodular lesion on a woman's earlobe. Actas Dermosifiliogr. 2013;104(5):437-478. http://dx.doi.org/10.1016/j.adengl. 2013. 04. 008

3. Doyle, L. A., Fletcher, C. D. M. Metastasizing "Benign” Cutaneous Fibrous Histiocytoma. Am J Surg Pathol. 2013; 37(4): 484-495.

4. Das, A., Das, A., Bandyopadhyay, D., Mishra, V., Saha, A. Aneurysmal benign fibrous histiocytoma presenting as a giant acrochordon on thigh. Indian Dermatol Online J. 2015; 6: 436-438.

5. Shin, J. W., Park, H.S., Kim, B. K., Kim, Y. A., Kim, M. G., Won, C. H. Aneurysmal benign fibrous histiocytoma with atrophic features. Ann Dermatol. 2009; 21(1): 42-45. 